\title{
Facial width-to-height ratio relates to dominance style in the genus Macaca
}

Marta Borgi, Bonaventura Majolo

Background. Physical, visual, chemical, and auditory cues signalling fighting ability have independently evolved in many animal taxa as a means to resolve conflicts without escalating to physical aggression. Facial width-to-height ratio (fWHR, i.e. the relative width to height of the face) has been associated with dominance-related phenotypes both in humans and in other primates. In humans, faces with a larger fWHR are perceived as more aggressive. Methods. We examined fWHR variation among 11 species of the genus Macaca. Macaques have been grouped into four distinct categories, from despotic to tolerant, based on their female dominance style. Female dominance style is related to intra- and inter-sexual competition in both males and females and the result of different evolutionary pressure across species. We used female dominance style as a proxy of intra/inter-sexual competition to test the occurrence of correlated evolution between competitive regimes and dominance-related phenotypes. fWHR was calculated from 145 2D-photographs of male and female adult macaques. Results. We found no phylogenetic signal on the differences in fWHR across species in the two sexes. However, fWHR was greater, in females and males, in species characterised by despotic female dominance style than in tolerant species. Discussion. Our results suggest that dominance-related phenotypes are related to differences in competitive regimes and intensity of inter- and intra-sexual selection across species. 
1 Facial width-to-height ratio relates to dominance style in the genus Macaca

4 Marta Borgi ${ }^{a, b}$ and Bonaventura Majolo ${ }^{a}$

5

6 aSchool of Psychology, University of Lincoln, Lincoln LN6 7TS, Great Britain

7 bSection of Behavioural Neuroscience, Department of Cell Biology and Neurosciences, Istituto

8 Superiore di Sanità, Rome, Italy

\section{Corresponding author:}

14 Marta Borgi

15 Section of Behavioral Neuroscience

16 Department of Cell Biology and Neurosciences

17 Istituto Superiore di Sanità

18 Viale Regina Elena 299, I-00161

19 Rome, Italy

20 Tel: +3906 49902107

21 Fax: +39064957821

22 E-mail: marta.borgi@iss.it 


\section{ABSTRACT}

25 Background. Physical, visual, chemical, and auditory cues signalling fighting ability have independently evolved in many animal taxa as a means to resolve conflicts without escalating to physical aggression. Facial width-to-height ratio (fWHR, i.e. the relative width to height of the face) has been associated with dominance-related phenotypes both in humans and in other primates. In humans, faces with a larger fWHR are perceived as more aggressive.

30 Methods. We examined fWHR variation among 11 species of the genus Macaca. Macaques

31 have been grouped into four distinct categories, from despotic to tolerant, based on their female

32 dominance style. Female dominance style is related to intra- and inter-sexual competition in both

33 males and females and the result of different evolutionary pressure across species. We used

34 female dominance style as a proxy of intra-/inter-sexual competition to test the occurrence of 35 correlated evolution between competitive regimes and dominance-related phenotypes. fWHR 36 was calculated from 145 2D-photographs of male and female adult macaques.

37 Results. We found no phylogenetic signal on the differences in fWHR across species in the two 38 sexes. However, fWHR was greater, in females and males, in species characterised by despotic 39 female dominance style than in tolerant species.

40 Discussion. Our results suggest that dominance-related phenotypes are related to differences in 41 competitive regimes and intensity of inter- and intra-sexual selection across species. 


\section{INTRODUCTION}

43 In animals, aggressive conflicts may bear significant fitness costs for the opponents (e.g. chronic

44 stress, severe injuries, limited access to resource, House et al. 1988), that can potentially out-

45 weigh the benefits of winning a conflict. Because of such potential costs, visual, chemical, tactile

46 and auditory cues, as well as physical traits, have independently evolved in various taxa to signal

47 the fighting ability of opponents and to allow animals to resolve conflicts without escalating to

48 physical aggression (Arnott \& Elwood 2009; Parker \& Rubenstein 1981). For example, animals

49 can maintain their dominance rank in a social group by signals of dominance/submission and

50 displacements that do not involve overt aggression (Preuschoft \& van Schaik 2000).

51 In humans, facial phenotype, specifically the facial width-to-height ratio (fWHR), is a cue of

52 aggression, dominance and fighting ability: fWHR is positively related to the acquisition of

53 status and resources, antisocial tendencies, dominance status and propensity to be aggressive

54 (Carré \& McCormick 2008; Carré et al. 2009; Haselhuhn \& Wong 2012; Lefevre et al. 2014a;

55 Sell et al. 2009). Preliminary evidence on the association between circulating testosterone and

56 fWHR in men (Lefevre et al. 2013), as well as the association between individual differences in

57 amygdala reactivity, fWHR and self-reported aggression (Carré et al. 2013), suggests a possible

58 path through which fWHR may have evolved to signal aggressive attitude. The association

59 between facial phenotype and aggression might reflect the common influence of pubertal

60 testosterone on cranial growth and the development of neural circuitry underlying aggressive

61 behaviour (Carré \& McCormick 2008; Carré et al. 2013). High fWHR may thus work as a

62 marker of "masculine" tendencies, in particular in species with a sex-biased frequency of

63 aggression. Previous studies on humans indicate that the correlation between fWHR and

64 dominance-related behaviours, as well as the modulator effect of fWHR on the relationship 
65 between amygdala reactivity and self-reported aggression, are either specific to or more robust in 66 men (Carré \& McCormick 2008; Carré et al. 2013; Geniole et al. 2014; Goetz et al. 2013;

67 Haselhuhn \& Wong 2012; Stirrat \& Perrett 2010; but see: Lefevre et al. 2014a).

68 Similarly to what happens in humans, non-human primates rely extensively on non-verbal communication and on a variety of facial displays to signal aggression/submission and to modulate social interactions (Maestripieri 1997). Therefore, facial phenotypes, such as the fWHR, can have a homologous function and a shared phylogenetic history in non-human primates and in the human lineage. In support to this hypothesis, two recent studies have shown that, in brown capuchin monkeys (Sapajus apella) fWHR is positively related to alpha status and to a dominance-related personality trait (Lefevre et al. 2014b; Wilson et al. 2014).

To date, research on the relationship between fWHR and behaviour has rarely been conducted on non-humans and it has mostly focused on within-species variation. If fWHR is a signal of aggression and fighting abilities, we predict correlated evolution between fWHR and social traits at the species level. In other words, we predict different evolutionary pressure on fWHR depending on the species-specific differences in aggressiveness, competitive regime and

80 dominance style. We tested this hypothesis in the genus Macaca, comprising 22 species with

81 similar group composition (i.e. multimale-multifemale social groups) but differing in female 82 dominance style and intra-group social relationships, according to a four-grade scale ranging 83 from despotic (grade 1) to tolerant (grade 4) (Balasubramaniam et al. 2012a; Balasubramaniam 84 et al. 2012b; Thierry 2000; Thierry et al. 2008; Thierry et al. 2000). Despotic, grade 1, species 85 are characterised by steep linear dominance hierarchies among females, whereby the outcome of 86 dyadic competitive interactions, as well as access to resources, strongly depend on the 87 dominance rank of the contestants (Balasubramaniam et al. 2012a; Balasubramaniam et al. 
88 2012b; Thierry 2000). Competitive interactions can quickly escalate into overt aggression and

89 result in injuries if a subordinate animal does not display submission to a dominant individual

90 (Thierry et al. 2000). In tolerant, grade 4, species, dominance hierarchies are shallow among

91 females, that is, the outcome of dyadic competitive interactions depends on context as much as it

92 does on the dominance rank of the contestants (Balasubramaniam et al. 2012a; Balasubramaniam

93 et al. 2012b; Thierry 2000). Low intensity aggressive interactions and counter-aggression are

94 more frequent in tolerant species than in despotic species, but conflicts less frequently result in

95 injuries, and access to resources is less rank-dependent (Balasubramaniam et al. 2012a;

96 Balasubramaniam et al. 2012b; Thierry 2000; Thierry et al. 2008; Thierry et al. 2000). Species

97 in-between the two extremes of this grading system (grade 2 and 3), show a mixture of 98 dominance style traits of tolerant and despotic species (Balasubramaniam et al. 2012a).

99 Differences in dominance style across macaque species have a strong phylogenetic signal

100 (Balasubramaniam et al. 2012a; Balasubramaniam et al. 2012b).

101 In female macaques, we predict correlated evolution between dominance-related phenotypes and

102 species-specific differences in dominance style: fWHR should be greater in females from

103 despotic species than in those from tolerant species. In despotic species, displays of dominance,

104 aggression and submission among females should be more important than in tolerant species as

105 in the former the risk of escalated aggression is expected to be higher. Over evolutionary time,

106 therefore, there should have been stronger pressure for the evolution of dominance-related

107 phenotypes in female macaques in despotic species than in tolerant species, other things being

108 equal (relative dominance rank of the individuals). We predict a significant relationship between

109 fWHR and female dominance style across species in male macaques. However, we cannot

110 predict whether such relationship is positive or negative, as it is currently not known if male 
111 macaques show a similar or opposite pattern of inter-specific differences in dominance style as

112 observed in females. Our prediction for male macaques is based on the fact that competitive

113 regimes among females are related to male-male competition and dominance style (Schulke \&

114 Ostner 2008; Schulke \& Ostner 2012) and can affect intra- and inter-sexual selection in both

115 sexes. For example, male reproductive skew is more pronounced in female tolerant species

116 (Schulke \& Ostner 2008; Schulke \& Ostner 2012; Van Noordwijk \& Van Schaik 2004). To test

117 our predictions we used the four-grade scale of dominance style in female macaques as a proxy

118 of differences across species in the level of inter- and intra-sexual selection in the two sexes. We

119 calculated fWHR from 145 two-dimensional images (a measure previously shown to correlate

120 with 3D scans and facial anthropometry, Kramer et al. 2012) of male/female faces of macaques

121 from 11 species representing the four grades of female dominance style - as in Thierry's

122 classification (2000). We analysed the correlation between fWHR and female dominance style

123 using phylogenetic-controlled analyses and standard (i.e. without phylogenetic control) multiple

124 regression.

125

126 MATERIALS \& METHODS

127 Images and measurements

128 We collected frontal images of the face of as many macaque species as possible following two

129 different approaches. First, we used pictures taken by one of us (BM) and requested pictures

130 taken by colleagues working on macaques in the wild or in captivity. Second, in order to expand

131 our sample size in terms of number of pictures and species, we searched for images on the

132 Google Images web search engine by submitting the scientific name of each species as a key-

133 word. In order to be included in our dataset, images had to be frontal, full-faced photographs 
134 depicting male and female adult macaques (i.e. $\geq 5$ years old for females and $\geq 7$ years old for

135 males) with a neutral expression (closed mouth) and with a resolution of at least $400 \times 300$ pixels

136 per inch. For the images collected from the web, information on the sex and age of the animal in

137 the image was often not available (sex and age of the animals was known for images taken by

138 BM or obtained from colleagues). Therefore, these images were independently scored for age

139 and sex by one of us (BM) and an expert on macaques (who was unaware of the aims of the

140 study). Correlation between the two scores was positive and significant (Spearman correlation;

141 age rho=0.70, $<<0.001$; sex: rho $=0.57, \mathrm{p}<0.001)$. However, we discarded all of the images for

142 which scores for sex and/or age were in disagreement between the two scorers in order to avoid

143 biasing the analyses due to incorrect data on the sex/age of the animals. Moreover, since

144 macaques often present individual physical traits which are easily identifiable, all images taken

145 from the web were checked for independence by two researchers, in order to reduce the risk that

146 the same animal could be depicted in two or more images included in the dataset (if that was the

147 case, we only kept one image, with the highest resolution). Following this procedure, our dataset

148 comprised a total of 145 images from 11 species of genus Macaca, 73 images of adult female

149 macaques (40 images collected from the web) and 72 of adult male macaques (41 images

150 collected from the web; Table 1).

151 Using Adobe Photoshop (Adobe Systems, San Jose, California), pictures were digitized at 72 dpi

152 and were two-dimensionally rotated (horizontally aligned) and scaled to the same inter-pupillary

153 distance, in order to standardise face size and head position across images. Facial measurements

154 were taken using the ruler tool with pixels as a unit. In accordance with previous work (Kramer

155 et al. 2012; Lefevre et al. 2014b; Wilson et al. 2014), fWHR was calculated by dividing the bi-

156 zygomatic width (maximum horizontal distance from the left to the right zygion) by the upper- 
157 face height (vertical distance from the highest point of the eyelids to the upper-lip) (Figure 1).

158 One of us (MB) took all of the measures; at the time of taking the measures MB was blind to the

159 dominance style of the different macaque species in the dataset. In order to test the consistency

160 of the facial measures taken across time, MB re-took the measures for the bi-zygomatic width

161 and the upper-face height for a subset of images $(\mathrm{N}=39)$, randomly selected for each species

162 (2.4 images per species), six months after the same measures were first taken on those images.

163 Consistency across the two measures was very high (Spearman correlation; bizygomatic width:

164 rho=0.93, $<<0.001$; upper-face height: $r h o=0.99, \mathrm{p}<0.001)$.

165

166 Data Analysis

167 Each species included in our dataset was assigned to one of the four grades of dominance style

168 (from 1=despotic up to $4=$ tolerant species; Table 1) following the classification of macaque

169 species available in Thierry 2000 (Table 6.2, p.112). We square-root transformed fWHR

170 (dependent variable) to improve normality and used two distinct analytical approaches to test our

171 hypothesis. First, we averaged the square-root transformed fWHR values per species and sex and

172 ran a phylogenetically controlled generalized least square regression model (PGLS)

173 independently for male and female macaques. Since the number of images available on each

174 species and sex varied significantly in our dataset (Table 1), we entered the number of images

175 for females (or males) as a control variable in the two PGLSs. We estimated Pagel's lambda

176 (Pagel, 1999) using maximum likelihood. In the results we present the estimated lambda for each

177 model and the $\mathrm{p}$ values of the likelihood ratio tests comparing the estimated lambda to the upper

178 (lambda $=1$ ) and lower bounds (lambda $=0$ ). We ran these two PGLSs in $\mathrm{R}$ 3.1.0 (R

179 Development Core Team 2011), using the CAPER 0.4 package (Orme et al. 2012). We 
180 downloaded a consensus phylogenetic tree, with the chronogram branch option using the

181 Genbank taxonomy, from the 10ktree primate phylogeny version 2

182 (http://10ktrees.fas.harvard.edu, Arnold et al. 2010). Second, since no phylogenetic signal was

183 detected in the two PGLSs (see results), we ran a standard (i.e. without phylogenetic control)

184 linear mixed model on the two sexes together. The fWHR values of each image (our dependent

185 variable) were our data points $(\mathrm{N}=145)$; dominance style and sex (females or males) were

186 entered as fixed factors, species ID was our random factor. We ran this linear mixed model using

187 Stata v.12.1 (Stata Corp 2011).

188

189 RESULTS

190 We had no significant result for the predicting variables in the two PGLSs run separately on

191 females and males; the two full models were also not significant (PGLS on females: $\mathrm{F}(2,8)=$

192 2.01, adjusted R-squared $=0.17, \mathrm{~N}=11, \mathrm{p}=0.20$; PGLS on males: $\mathrm{F}(2,8)=3.21$, adjusted R-

193 squared $=0.31, \mathrm{~N}=11, \mathrm{p}=0.10)$. Dominance style was negatively related to fWHR in the two

194 sexes (i.e. fWHR was greater in despotic than in tolerant species) but this relationship was not

195 significant (females: coefficient $\pm \mathrm{SE}=-0.02 \pm 0.01, \mathrm{t}=-1.69, \mathrm{p}=0.13$; males: coefficient $\pm \mathrm{SE}$

$196=-0.02 \pm 0.01, \mathrm{t}=-1.71, \mathrm{p}=0.13$; Figure 2). The number of images available for each species

197 and sex did not have a significant effect in any of the two PGLSs (females: coefficient $\pm \mathrm{SE}=-$

$1980.01 \pm 0.01, \mathrm{t}=-1.20, \mathrm{p}=0.26$; males: coefficient $\pm \mathrm{SE}=0.01 \pm 0.01, \mathrm{t}=1.19, \mathrm{p}=0.09)$. Pagel's

199 lambda values (Pagel, 1999) were equal to zero in the two PGLSs on males (likelihood ratio test:

200 upper bound $p=1.00$; lower bound $p=0.12$ ) and females (upper bound $p=1.00$; lower bound $p$

$201<0.01$ ), indicating a weak phylogenetic signal for the relationship between fWHR and female 202 dominance style. 
203 A linear mixed model on the effect of female dominance style and sex of the animal, as fixed

204 factors, on fWHR (species ID entered as a random factor) showed that fWHR was significantly

205 greater in despotic species than in tolerant species (coefficient $\pm \mathrm{SE}=-0.20 \pm 0.01, \mathrm{z}=-1.90, \mathrm{p}<$

206 0.05; Figure 3). However, fHWR did not significantly differ between female and male macaques

207 (coefficient $\pm \mathrm{SE}=-0.00 \pm 0.01, \mathrm{z}=-0.09, \mathrm{p}=0.93$ ).

208

209 DISCUSSION

210 Our study is the first to analyse the relationship between species-specific differences in

211 dominance style and fWHR. The four-grade scale of female dominance style was significantly

212 related to fWHR (female despotic species having greater fWHR than tolerant species) in male

213 and female macaques, but not when fWHR was averaged per species and sex. Moreover, we

214 found no phylogenetic signal for differences in fWHR across macaque species.

215 fWHR is a signal of aggression and fighting ability that might facilitate the resolution of

216 conflicts of interest without the need for these to escalate into overt aggression (Carré \&

217 McCormick 2008; Carré et al. 2009; Lefevre et al. 2014a; Sell et al. 2009). As such, fWHR may

218 affect decision-making in competitive interactions (e.g. fight or flight) and can minimize the

219 costs of competition to contestants for both won/lost and unresolved conflicts, including chronic

220 stress, severe injuries and deaths (Arnott \& Elwood 2009; Blanchard et al. 2011). In humans,

221 fWHR predicts aggressive behaviour (e.g. Carré \& McCormick 2008; Goetz et al. 2013),

222 especially in males, and it may operate as a signal of physical dominance evolved under sexual

223 selection (Weston et al. 2004; Weston et al. 2007). Our study suggests that fWHR may have a

224 similar function and be related to sexual selection and sexual dimorphism in non-human primates 
225 (Lefevre et al. 2014b; Leutenegger \& Kelly 1977; Plavcan \& van Schaik 1992; Wilson et al. 226 2014).

227 Analyses on female macaques support our prediction that species-specific differences in 228 dominance style are related to fWHR, possibly because physical and behavioural traits have been 229 under similar evolutionary pressure (Balasubramaniam et al. 2012a; Balasubramaniam et al. 230 2012b; Thierry 2000; Thierry et al. 2008). We found a similar pattern in male macaques.

231 However, the interpretation of our findings in males is difficult, since scarce data are available

232 on dominance style difference across species in male macaques and on how such differences are

233 related to female dominance style. Male reproductive skew is thought to be higher in species

234 where female-female relationships are classified as being tolerant than in species where female-

235 female relationships are despotic (Schulke \& Ostner 2008; Schulke \& Ostner 2012), other things

236 being equal (e.g. operational sex ratio). Male reproductive skew is positively related to the

237 degree of paternal relatedness in a species (Ostner et al. 2008; Widdig 2013) but negatively

238 related to female oestrous synchrony and mate choice (Dubuc et al. 2011; Soltis et al. 1997).

239 Because of the limited data on male-male relationships, a parsimonious interpretation of our

240 findings is that differences in female dominance style are related to species-specific differences

241 in intra- and inter-sexual selection in the two sexes. Female dominance style can be used as a

242 proxy of inter-specific differences in selection and competitive regimes. However, the exact

243 nature of the cause-effect relationship between male fWHR and dominance style differences in

244 males and females cannot be analysed until data are available. Because of the scarcity of data on

245 males, since our results from phylogenetic analyses and multiple regression differed in their

246 significance level (this could be due, at least partially, to the small number of species available in

247 our dataset) and given that we cannot completely rule out the possibility that we had in the 
248 dataset more than one image for each animal (see methods), our findings have to be interpreted

249 with caution.

250 Three areas of research require additional data and study testing alternative hypotheses. First, we

251 know very little on the developmental trajectory of fWHR, the role of hormones, of the brain and

252 of sexual maturation. In humans and other anthropoid primates, fWHR has been described as a

253 sexually dimorphic trait that arises around puberty (coincident with the rise in pubertal

254 testosterone) and that is, at least to some extent, not explained by sex differences in body size

255 (Carré \& McCormick 2008; Weston et al. 2004; Weston et al. 2007). Preliminary evidence has

256 shown a positive correlation between fWHR and circulating testosterone in men (Lefevre et al.

257 2013). Moreover, the association between fWHR and dominance-related behaviour may be more

258 evident among individuals low in status (Carré 2014; Goetz et al. 2013; Welker et al. 2015). If so,

259 life-history variables such as age at sexual maturation, degree of social instability (e.g. frequency

260 of rank reversals) and of stress (due to competition, e.g. Crockford et al. 2008) could inter-play

261 with dominance rank in affecting fWHR throughout an animal's life.

262 Second, intra-species variance across populations/groups in dominance style is expected to be

263 high but the causes of behavioural flexibility are still little understood (Kamilar \& Baden 2014).

264 The scarcity of images of macaque faces for which data were also available on the dominance

265 rank of each animal, as well as on individual- and group-specific social traits (e.g. conciliatory

266 tendency, steepness of the hierarchy or frequency of counter-aggression), forced us to enter in

267 the analyses the four-grade system of dominance style instead of more specific measures of

268 social style. Ideally, additional data are needed to analyse the relative role of each social trait

269 contributing to the species/population/individual dominance style, the degree of inter- and intra- 
270 species variation in fWHR, and to what extent such variation is explained by shared phylogenetic

271 history or current socio-ecological factors (e.g. level of competition in a group).

272 A third limitation of our study, and an area that requires further investigation, is whether two-

273 dimensional images used to calculate facial measurements reliably 'represent' how non-human

274 primates see faces of their conspecifics, especially in the case of pictures taken in the absence of 275 controlled conditions.

276

277 CONCLUSIONS

278 In conclusion, our research makes a novel contribution to the study of dominance-related

279 phenotypes, by showing that fWHR is related with female dominance style in male and female 280 macaques. This study has to be considered as one of the first steps towards understanding 281 whether and how sexual selection, socio-ecological variables, reproductive strategies and life282 history variables affect dominance-related phenotypes.

283

284

285

286

287

288

289

290

291

292

\section{ACKNOWLEDGEMENTS}

We would like to thank Rebecca Ayre for help to collect the images used in this study. We are extremely grateful to Simone Giancontieri and Christopher Young for their help with the fWHR measures and data analysis, and to friends and colleagues who shared their images of macaques with us. We would like to thank Gabriele Schino for useful comments on our study. We are extremely grateful to Lauren Brent and the Caribbean Primate Research Center for sharing their pictures of Macaca mulatta. We would also like to thank Emily Bethell, Jerome Micheletta and 
293 Bernard Thierry, for kindly agreeing to share their images of macaques with us, Juliane

294 Kaminski, Jerome Micheletta and James Higham for very useful comments on previous drafts of 295 our manuscript.

296

297

298

299

300

301

302

303

304

305

306

307

308

309

310

311

312

313

314

315

316

317

318

319

320

321

322

323

324

325

326

327

\section{REFERENCES}

Arnold C, Matthews L, and Nunn C. 2010. The 10kTrees Website: A new online resource for primate phylogeny. Evol Anthropol 19:114-118. 10.1002/evan.20251

Arnott G, and Elwood R. 2009. Assessment of fighting ability in animal contests. Anim Behav 77:991-1004. 10.1016/j.anbehav.2009.02.010

Balasubramaniam K, Dittmar K, Berman C, Butovskaya M, Cooper M, Majolo B, Ogawa H, Schino G, Thierry B, and de Waal F. 2012a. Hierarchical steepness and phylogenetic models: phylogenetic signals in Macaca. Anim Behav 83:1207-1218. 10.1016/j.anbehav.2012.02.012

Balasubramaniam KN, Dittmar K, Berman CM, Butovskaya M, Cooper MA, Majolo B, Ogawa H, Schino G, Thierry B, and De Waal FB. 2012b. Hierarchical steepness, counteraggression, and macaque social style scale. Am $J$ Primatol 74:915-925. 10.1002/ajp. 22044

Blanchard DC, Griebel G, Pobbe R, and Blanchard RJ. 2011. Risk assessment as an evolved threat detection and analysis process. Neurosci Biobehav Rev 35:991-998. 10.1016/j.neubiorev.2010.10.016

Carré JM. 2014. Social status, facial structure, and assertiveness in brown capuchin monkeys. Front Psychol 5:567. doi: 10.3389/fpsyg.2014.00567.

Carré JM, and McCormick CM. 2008. In your face: facial metrics predict aggressive behaviour in the laboratory and in varsity and professional hockey players. Proc Biol Sci 275:26512656. 10.1073/pnas.0705435104

Carré JM, McCormick CM, and Mondloch CJ. 2009. Facial structure is a reliable cue of aggressive behavior. Psychol Sci 20:1194-1198. 10.1111/j.1467-9280.2009.02423.x

Carré JM, Murphy KR, and Hariri AR. 2013. What lies beneath the face of aggression? Soc Cogn Affect Neurosci 8:224-229. 10.1093/scan/nsr096

Crockford C, Wittig RM, Whitten PL, Seyfarth RM, and Cheney DL. 2008. Social stressors and coping mechanisms in wild female baboons (Papio hamadryas ursinus). Horm Behav 53:254-265. 10.1016/j.yhbeh.2007.10.007

Dubuc C, Muniz L, Heistermann M, Engelhardt A, and Widdig A. 2011. Testing the priority-ofaccess model in a seasonally breeding primate species. Behav Ecol Sociobiol 65:16151627. 
328 Geniole S, Keyes A, Carré J, and McCormick C. 2014. Fearless dominance mediates the

329

330

331

332

333

334

335

336

337

338

339

340

341

342

343

344

345

346

347

348

349

350

351

352

353

354

355

356

357

358

359

360

361

362

363

364

365

366

367 relationship between the facial width-to-height ratio and cheating. Pers Individ Dif 57:5964. 10.1016/j.paid.2014.05.019

Goetz SM, Shattuck KS, Miller RM, Campbell JA, Lozoya E, Weisfeld GE, and Carre JM. 2013. Social status moderates the relationship between facial structure and aggression. Psychol Sci 24:2329-2334. 10.1177/0956797613493294

Haselhuhn MP, and Wong EM. 2012. Bad to the bone: facial structure predicts unethical behaviour. Proc Biol Sci 279:571-576. 10.1098/rspb.2011.1193

House JS, Landis KR, and Umberson D. 1988. Social relationships and health. Science 241:540545. 10.1126/science.3399889

Kamilar J, and Baden A. 2014. What drives flexibility in primate social organization? . Behav Ecol Sociobiol 68:1677-1692. doi:10.1007/s00265-014-1776-x

Kramer RS, Jones AL, and Ward R. 2012. A lack of sexual dimorphism in width-to-height ratio in white European faces using 2D photographs, 3D scans, and anthropometry. PLoS One 7:e42705. 10.1371/journal.pone.0042705

Lefevre C, Lewis G, Perrett D, and Penke L. 2013. Telling facial metrics: facial width is associated with testosterone levels in men. Evol Hum Behav 34:273-279. 10.1016/j.evolhumbehav.2013.03.005

Lefevre CE, Etchells PJ, Howell EC, Clark AP, and Penton-Voak IS. 2014a. Facial width-toheight ratio predicts self-reported dominance and aggression in males and females, but a measure of masculinity does not. Biol Lett 10:20140729. 10.1098/rsbl.2014.0729

Lefevre CE, Wilson VA, Morton FB, Brosnan SF, Paukner A, and Bates TC. 2014b. Facial width-to-height ratio relates to alpha status and assertive personality in capuchin monkeys. PLoS One 9:e93369. 10.1371/journal.pone.0093369

Leutenegger W, and Kelly JT. 1977. Relationship of sexual dimorphism in canine size and body size to social, behavioral, and ecological correlates in anthropoid primates. Primates, 18(1):117-136.

Maestripieri D. 1997. Gestural communication in macaques: usage and meaning of nonvocal signals. Evolution of communication 1:193-222. 10.1075/eoc.1.2.03mae

Orme C, Freckleton R, Thomas G, Petzoldt T, Fritz S, and Isaac N. 2012. CAPER: comparative analyses of phylogenetics and evolution in R. Package version 0.5. ed.

Ostner J, Nunn CL, and Schulke O. 2008. Female reproductive synchrony predicts skewed paternity across primates. Behav Ecol 19:1150-1158. 10.1093/beheco/arn093

Pagel M. 1999. Inferring the historical patterns of biological evolution. Nature, 401(6756): 877884. $10.1038 / 44766$

Parker G, and Rubenstein D. 1981. Role assessment, reserve strategy, and acquisition of information in asymmetric animal conflicts. Anim Behav 29:221-240. 10.1016/S00033472(81)80170-4

Plavcan JM, and van Schaik CP. 1992. Intrasexual competition and canine dimorphism in anthropoid primates. Am J Phys Anthropol 87(4):461-477. 10.1002/ajpa.1330870407 
368

369

370

371

372

373

374

375

376

377

378

379

380

381

382

383

384

385

386

387

388

389

390

391

392

393

394

395

396

397

398

399

400

401

402

403

404

405

406

Preuschoft S, and van Schaik C. 2000. Dominance and communication: Conflict management in various social settings. In: Aureli F, and de Waal FBM, eds. Natural conflict resolution. Berkeley: University of California Press, 106-128.

R Development Core Team. 2011. A language and environment for statistical computing. $\mathrm{R}$ Foundation for Statistical Computing. Vienna.

Schulke O, and Ostner J. 2008. Male reproductive skew, paternal relatedness, and female social relationships. Am J Primatol 70:695-698. 10.1002/ajp.20546

Schülke O, and Ostner J. 2012. Ecological and social influences on sociality. In: Mitani J, Call J, Kappeler P, Palombit R and Silk J, eds. The Evolution of Primate Societies. Chicago: University of Chicago Press, 193-219.

Sell A, Cosmides L, Tooby J, Sznycer D, von Rueden C, and Gurven M. 2009. Human adaptations for the visual assessment of strength and fighting ability from the body and face. Proc Biol Sci 276:575-584. 10.1098/rspb.2008.1177

Soltis J, Mitsunaga F, Shimizu K, Nozaki M, Yanagihara Y, Domingo-roura X, and Takenaka O. 1997. Sexual selection in Japanese macaques II: female mate choice and male-male competition. Anim Behav 54:737-746.

Stata Corp. 2011. Stata statistical software: Release 12. Texas: Stata Press.

Stirrat M, and Perrett DI. 2010. Valid facial cues to cooperation and trust: male facial width and trustworthiness. Psychol Sci 21:349-354. 10.1177/0956797610362647

Thierry B. 2000. Covariation of conflict management patterns across macaque species. In: Aureli F, and de Waal FBM, eds. Natural conflict resolution. Berkeley: University of California Press, 106-128.

Thierry B, Aureli F, Nunn CL, Petit O, Abegg C, and De Waal F. 2008. A comparative study of conflict resolution in macaques: insights into the nature of trait covariation. Anim Behav 75:847-860. 10.1016/j.anbehav.2007.07.006

Thierry B, Iwaniuk AN, and Pellis SM. 2000. The influence of phylogeny on the social behaviour of macaques (Primates: Cercopithecidae, genus Macaca). Ethology 106:713728. 10.1046/j.1439-0310.2000.00583.x.

Van Noordwijk M, and Van Schaik C. 2004. Sexual selection and the careers of primate males: paternity concentration, dominance-acquisition tactics and transfer decisions. In: Kappeler P, and Van Schaik C, eds. Sexual selection in primates: New and comparative perspectives. Cambridge: Cambridge University Press, 208.

Welker K, Goetz S, and Carré J. 2015. Perceived and experimentally manipulated status moderates the relationship between facial structure and risk-taking. Evol Hum Behav.

Weston EM, Friday AE, Johnstone RA, and Schrenk F. 2004. Wide faces or large canines? The attractive versus the aggressive primate. Proc Biol Sci 271 Suppl 6:S416-419. 10.1098/rsbl.2004.0203

Weston EM, Friday AE, and Lio P. 2007. Biometric evidence that sexual selection has shaped the hominin face. PLoS One 2:e710. 10.1371/journal.pone.0000710 
407 Widdig A. 2013. The Impact of male reproductive skew on kin structure and sociality in multi408 male groups. Evol Anthropol 22:239-250. 10.1002/evan.21366

409 Wilson VAD, Lefevre CE, Morton FB, Brosnan SF, Paukner A, and Bates TC. 2014. Personality 410 and facial morphology: Links to assertiveness and neuroticism in capuchins (Sapajus 411 [Cebus] apella). Pers Individ Dif 58:89-94. 10.1016/j.paid.2013.10.008

412 


\section{Table $\mathbf{1}$ (on next page)}

Table 1

Number of images used for the analyses and mean fWHR (square root transformed) divided by species, sex and dominance style 


\section{TABLES}

2 Table 1. Number of images used for the analyses and mean fWHR (square root transformed)

3 divided by species, sex and dominance style

\begin{tabular}{|c|c|c|c|c|c|c|c|}
\hline \multirow[b]{2}{*}{ Scientific } & \multirow[b]{2}{*}{ Common name } & \multirow{2}{*}{$\begin{array}{c}\text { Dominanc } \\
\text { e style* }\end{array}$} & \multicolumn{3}{|c|}{ Number of pictures } & \multicolumn{2}{|c|}{ Mean fWHR } \\
\hline & & & Femal & Mal & Tota & Femal & Male \\
\hline name & & & e & $\mathbf{e}$ & $\mathbf{l}$ & e & \\
\hline \multirow[t]{2}{*}{ M. cyclopis } & Formosan rock & & & & & & \\
\hline & macaque & 1 & 2 & 4 & 6 & 1.18 & 1.10 \\
\hline M. fuscata & Japanese macaque & 1 & 14 & 4 & 18 & 1.05 & 1.06 \\
\hline M. mulatta & Rhesus macaque & 1 & 9 & 11 & 20 & 1.13 & 1.15 \\
\hline M. nemestrina & Pig-tailed macaque & 2 & 2 & 12 & 14 & 1.04 & 1.05 \\
\hline M. fascicularis & Long-tailed macaque & 2 & 4 & 4 & 8 & 1.10 & 1.16 \\
\hline M. sinica & Toque macaque & 3 & 6 & 5 & 11 & 1.15 & 1.10 \\
\hline M. arctoides & Stump-tailed macaque & 3 & 5 & 4 & 9 & 1.08 & 1.09 \\
\hline M. sylvanus & Barbary macaque & 3 & 10 & 7 & 17 & 1.08 & 1.09 \\
\hline M. radiata & Bonnet macaque & 3 & 8 & 5 & 13 & 1.09 & 1.07 \\
\hline \multirow[t]{2}{*}{ M. nigra } & Crested black & & & & & & \\
\hline & macaque & 4 & 6 & 7 & 13 & 0.99 & 0.97 \\
\hline \multirow[t]{2}{*}{ M. tonkeana } & Tonkean macaque & 4 & 7 & 9 & 16 & 1.07 & 1.10 \\
\hline & & & 73 & 72 & 145 & & \\
\hline
\end{tabular}

4 * Category \#1 defines female despotic species - Grade \#1 in Thierry's classification (Thierry

5 2000) - and category \#4 defines female tolerant species - Grade \#4 in Thierry's classification 
1

Macaque faces

On the left. An example illustrating how fWHR was calculated from images (Barbary macaque). Bizygomatic width was measured as the horizontal distance between the left and right zygion (vertical lines); upper-face height as the vertical distance between the highest point of the eyelids and the highest point of the upper lip (horizontal lines). The fWHR was calculated as width divided by height. In the middle: a male rhesus macaque (dominance style 1). Photo by Lauren Brent (modified). On the right: a male Tonkean macaque (dominance style 4). Photo by Bernard Thierry (modified).
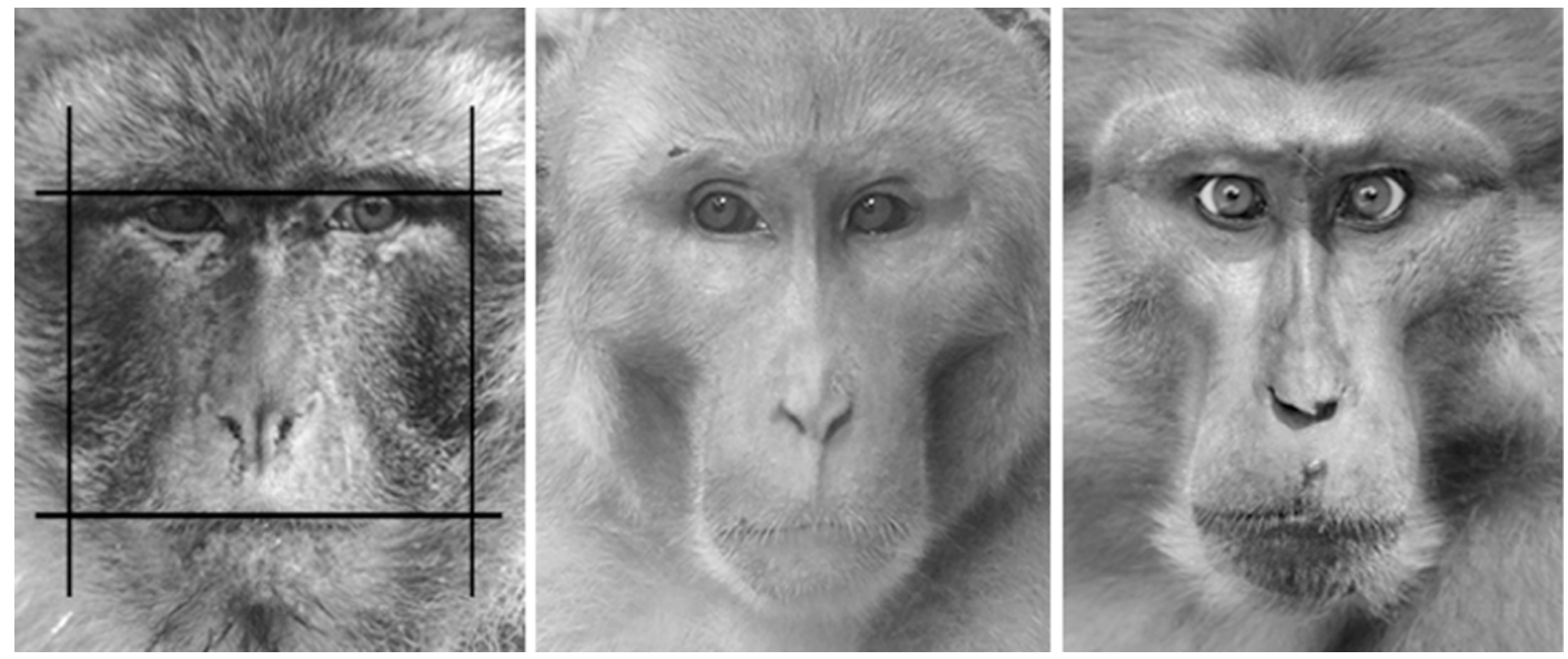
2

Relationship between female dominance style and fWHR (data averaged per species).

Scatter plot and line of best fit for the relationship between dominance style ( $y$ axis) and fWHR ( $x$ axis) in female (top) and male (bottom); grade 1 defines despotic species and grade 4 tolerant species (Thierry 2000).

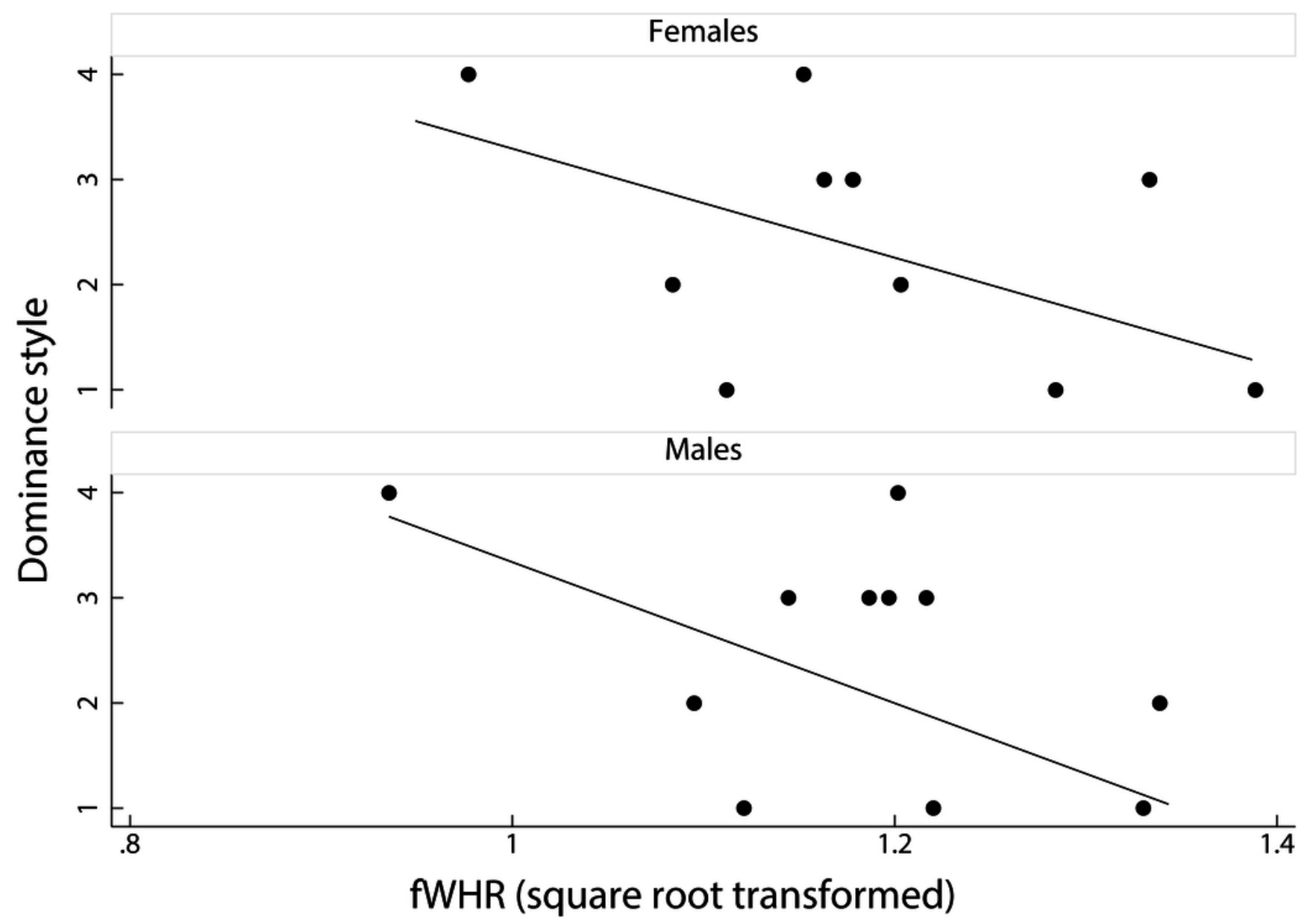


3

Relationship between female dominance style and fWHR (data points represent each image in the dataset).

Scatter plot and line of best fit for the relationship between dominance style (y axis) and fWHR ( $x$ axis) in female (top) and male (bottom); grade 1 defines despotic species and grade 4 tolerant species (Thierry 2000).

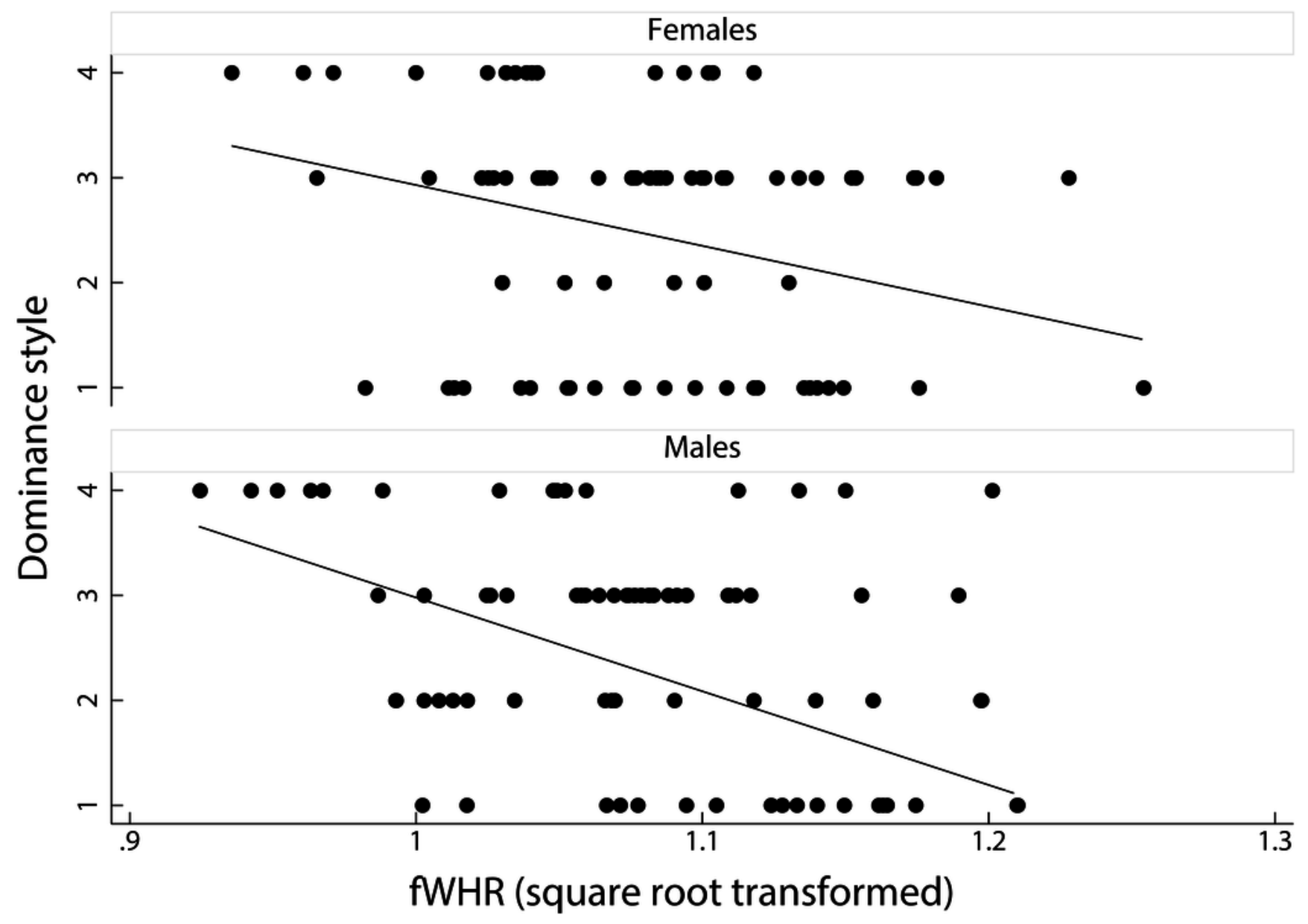

